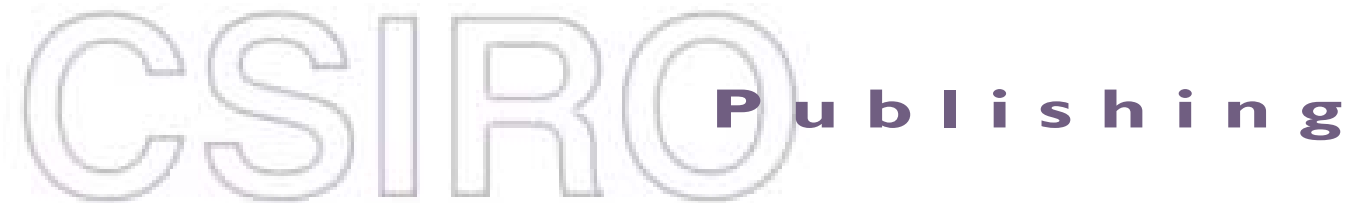

\section{Publications of the Astronomical Society of Australia}

Volume 19, 2002

(C) Astronomical Society of Australia 2002

An international journal of astronomy and astrophysics

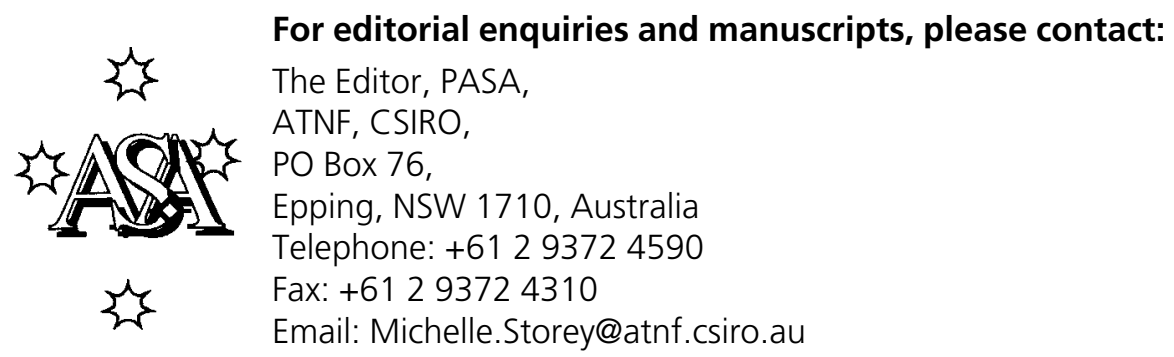

For general enquiries and subscriptions, please contact: CSIRO Publishing PO Box 1139 (150 Oxford St)

Collingwood, Vic. 3066, Australia

Telephone: +6139662 7666

Fax: +61 396627555

Email: publishing.pasa@csiro.au

C S I RO

PUBLISHING Published by CSIRO Publishing

for the Astronomical Society of Australia

www.publish.csiro.au/journals/pasa 


\title{
Millimetre Continuum Variations, VLBI Structure, and Gamma-rays: Investigating Shocked Jet Physics
}

\author{
E. Valtaoja ${ }^{1,2}$, T. Savolainen ${ }^{1}$, K. Wiik ${ }^{1,3}$ and A. Lähteenmäki ${ }^{3}$ \\ ${ }^{1}$ Tuorla Observatory, FIN-21500 Piikkiö, Finland \\ esko.valtaoja@utu.fi \\ ${ }^{2}$ Department of Physics, Turku University, FIN-20100 Turku, Finland \\ ${ }^{3}$ Metsähovi Radio Observatory, Helsinki University of Technology, FIN-02540 Kylmälä, Finland
}

Received 2001 August 1, accepted 2002 February 19

\begin{abstract}
We compare the total flux density variations and the VLBI structural variations in a sample of 27 gamma-ray blazars. We find that all the radio variations are due to shocks; the flux of the underlying jet remains constant. A large fraction of the shocks grow and fade within the innermost 0.1 mas, appearing only as 'core flares'. Comparisons with the EGRET data show that gamma-ray flares must come from the shocks, not from the jet. At the time of an EGRET flare, the shock is typically already over a parsec downstream from the radio core, beyond the accretion disk and/or the broad line region (BLR) photon fields. Thus, present models for gamma-ray production are inadequate, since they typically model the gamma-ray inverse Compton flux as coming from the jet, with significant disk or BLR external Compton components.
\end{abstract}

Keywords: galaxies: active — galaxies: jets — quasars: general — radio continuum: galaxies — techniques: interferometric

\section{Introduction}

In radio-bright active galactic nuclei (AGN) a fraction of the accretion energy and mass is transformed into a directed outflow. The ultimate goal is to understand the physics of these relativistic jets. Naturally, for radio-bright objects radio observations are crucial; however, radio is also the only regime in which we can obtain both images of the parsec-scale structure (with VLBI) and a fully sampled record of the activity (with total flux density monitoring). Radio data is therefore the key for understanding also the phenomena in other frequencies. In this paper we present results from comparisons between VLBI and total flux density (TFD) data, and demonstrate how these can be used for testing proposed models for gamma-ray production in AGN. We show that neglecting well-established results from the radio regime can lead to fundamentally flawed basic assumptions about the production of gamma rays. The results will be presented in more detail by T. Savolainen et al. and A. Lähteenmäki et al. (both in preparation).

\section{TFD Variations and Their Connection to VLBI Structural Changes}

Since the introduction of detailed shocked jet models (Marscher \& Gear 1985; Hughes, Aller, \& Aller 1985) the tacit assumption has been that the shocks forming in the flow are seen both as TFD radio flares and as new VLBI components emerging from the core (e.g. Valtaoja et al. 1988, 1992). However, hard evidence has been limited to a few individual cases: 3C 273 (Abraham et al. 1996; Türler, Courvoisier, \& Paltani 1999), 0528+134 (Krichbaum et al. 1998), 3C 345 (Valtaoja et al. 1999), PKS 0420-014 (Britzen et al. 2000), and 3C 279 (Wehrle et al. 2001). In order to reliably identify the new components and to resolve them both from the core and from each other, mm-VLBI monitoring is necessary, but large data sets have become available only recently. Jorstad et al. (2001a) monitored a sample of 42 gamma-ray bright blazars at 22 and $43 \mathrm{GHz}$ with the VLBA during 19931997. Of these, 27 have sufficient Metsähovi monitoring data (Teräsranta et al. 1998) at 22 and $37 \mathrm{GHz}$. We have shown earlier that all the TFD variations can to a surprising accuracy be modelled with a small number of exponential flares superposed on a constant baseline flux (Valtaoja et al. 1999). Figure 1 shows an example, 3C 279. We have now compared these flux decompositions with the VLBI component flux changes in the 27 common sources.

Our results confirm the expectations of the shocked jet models. Whenever a new VLBI component appears, the TFD decomposition shows a coinciding flare. The VLBI component fluxes correlate with the TFD flare fluxes (Pearson correlation coefficient 0.76; Spearman rank correlation coefficient 0.82 , with $\mathrm{P}=8 \times 10^{-14}$ ). Defining the 'beginning' of the exponential flare as $t_{\max }-\tau$, with $\tau$ the variability timescale, we also find that in 14 of the 17 cases the difference between the extrapolated VLBI zero epoch and the beginning of the TFD flare is $\leq 0.3$ years. The error in the estimated VLBI zero epoch is typically \pm 0.1 years or more, and the average frequency of large TFD flares is less than once a year; considering the uncertainties in the VLBI data and the difficulties in separating closely spaced TFD flares, the correlations are more than satisfactory. One example, $0528+134$, is shown in Figure 2.

The literature is full of cases where the VLBI radio core flux has been observed to change; these are commonly referred to as 'VLBI core flares', since the flux variations 

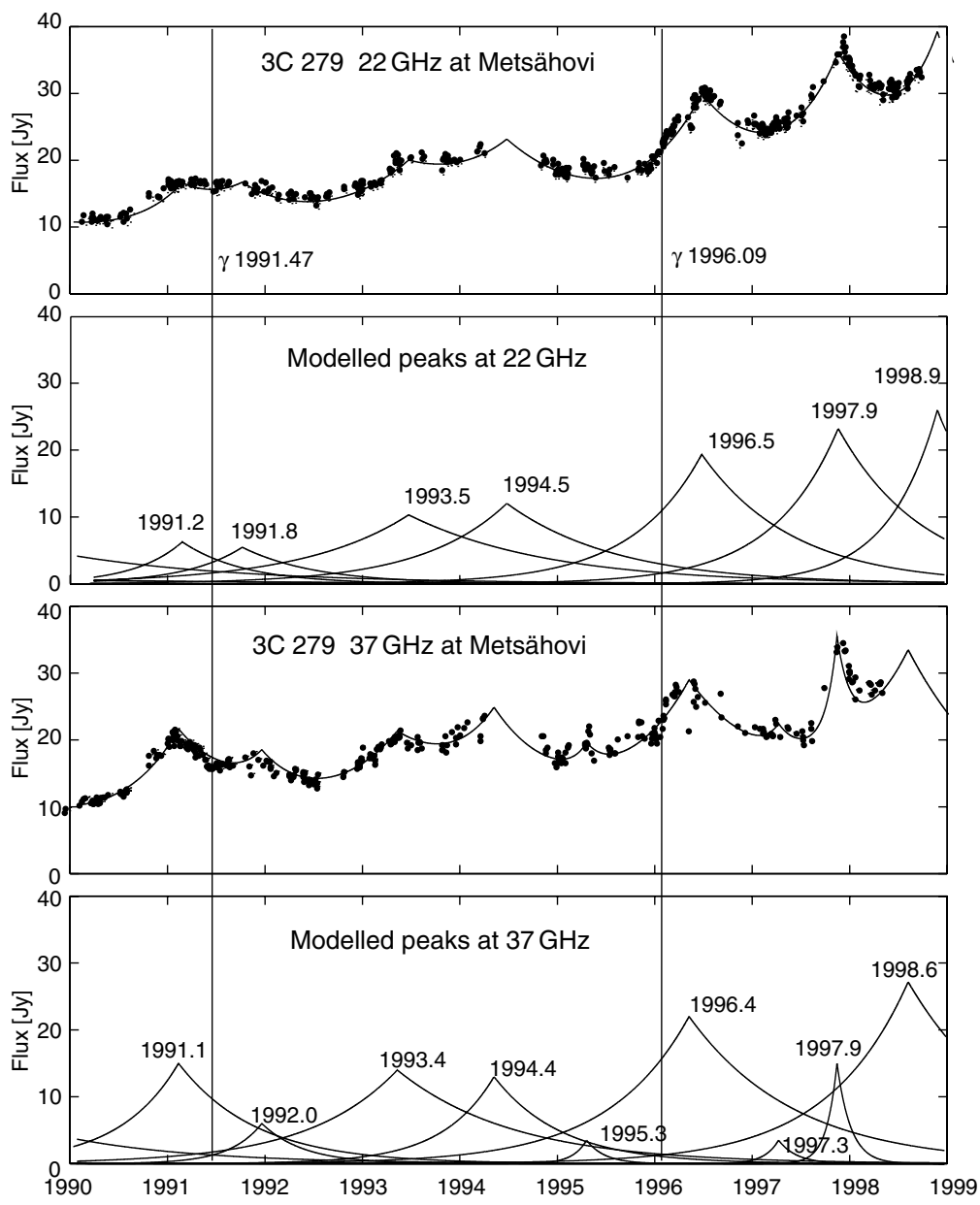

Figure 1 Metsähovi monitoring data for 3C 279 (dots) and exponential flare fits to the data. The two vertical lines show the epochs of the strongest EGRET gamma-ray flares in 1991.47 and 1996.09.

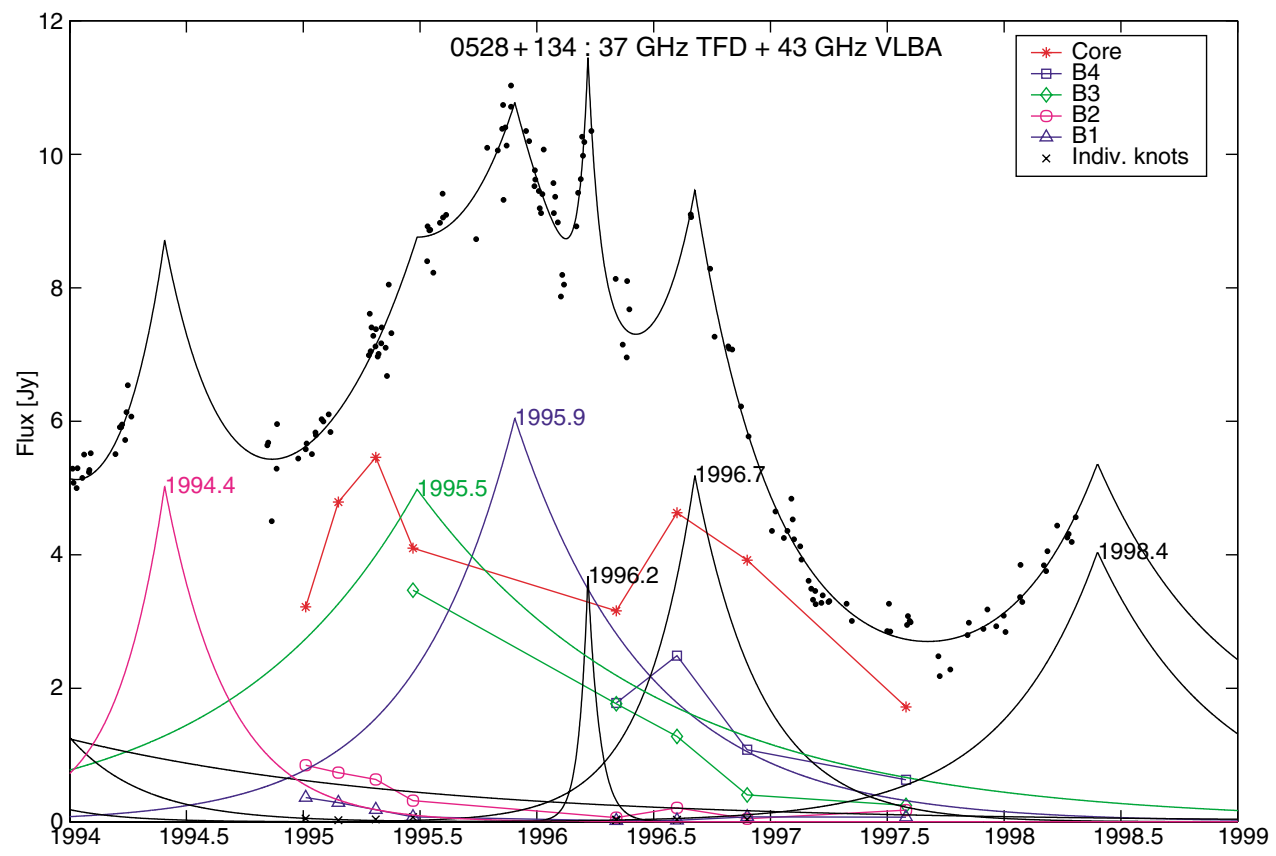

Figure 2 Metsähovi monitoring data for $0528+134$ and our exponential flare fits to the data, compared with the VLBI component flux variations. The symbols for the colour-coded VLBI components are identified in the box, using the notation of Jorstad et al. (2001a). The VLBI data points have been connected with straight lines to guide the eye. The smooth exponential curves show the TFD flare fits to the data, colour-coded to the corresponding VLBI components. Note how the VLBI components B1-B4 correspond to TFD flares. 
are similar to those associates with new VLBI components. To take just one example, in the recent $22 / 43 \mathrm{GHz}$ VLBA monitoring of 3C 279 between 1991 and 1997 (Wehrle et al. 2001), virtually all large TFD changes were due to changes in the VLBI core flux. Similar behaviour is common also in the Jorstad et al. (2001a) data (cf. Figure 2). The core flares are either related to true changes in the radio core, or they are due to VLBI components which blend with the radio core. In the first case the parameters of the underlying jet flow must change, resulting in enhanced radiation from its innermost parts. In the second case the shocks must grow and decay within $\approx 0.15$ mas (typical VLBA beamsize).

Since Jorstad et al. (2001a) have estimated the typical expansion speeds of the sources, we can estimate the separation between the true core and the new component during a core flare, if the second explanation is valid. We selected five core flares, in which the estimated separation during the VLBI observation was larger than a third of the beamsize, and reanalysed the Jorstad et al. (2001a) data by fitting two close circular Gaussian components to the core. In each case, the closure phases supported a second component with the predicted flux and separation (between 0.05 and 0.12 mas) from the core. Thus, core flares appear to be 'standard' shocks which fade below detection threshold already within one VLBA beamsize.

The conclusion from our study is clear: whenever radio flux rises, a new shock has been created in the jet. Judging from the TFD flux curves, the shock develops approximately as predicted in the standard Marscher \& Gear (1985) model. There is no evidence for the other possible explanation for flux variations, a change in the jet parameters such as the bulk Lorentz or the Doppler boosting factor, or the electron energy spectrum.

Of course, something must change in the flow to cause the formation of a new shock. However, the essence of shock models is that the observed flux changes are due to changes in the radio luminosity of the evolving shock, not in the luminosity of the underlying flow. The shock models are two-component models, the observed flux being the sum of a constant component (the flow) and a changing component (the shock).

\section{The Gamma-ray Connection}

The conclusion of the previous section is in itself nothing new (cf. Valtaoja et al. 1988), and has indeed been generally accepted as the standard framework by radio astronomers studying blazars. However, for models of high-frequency radiation it has wide-ranging implications, which have equally generally been ignored by gamma-ray theoreticians. Consider the state of the art in gamma-ray model fitting, 3C 279 (Hartman et al. 2001). The eleven radio to gamma spectra obtained between 1991 and 2000 have been quite well fitted using the model of Böttcher \& Bloom (2000), which calculates both the direct synchrotron component from the jet and the inverse Compton components: synchrotron self-Compton (SSC) with synchrotron seed photons, and external Compton (EC) with

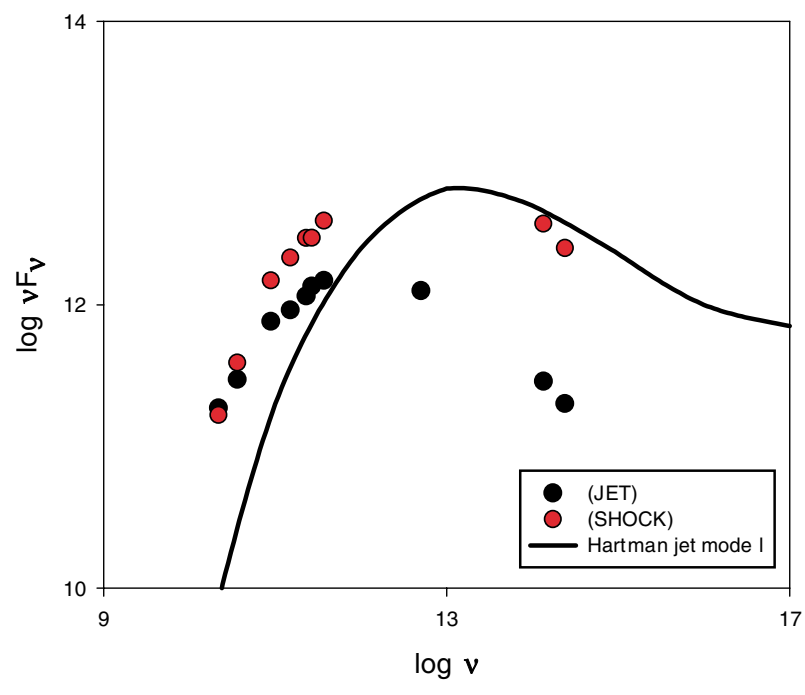

Figure 3 The estimated shock (variable) and jet (constant) components in 3C 279 at 1991.0 during a moderate radio flare, compared with assumed jet spectrum of Hartman et al. (2001) for the EGRET epoch 1991.47. (During 1991.47 the situation was more complicated, with two shocks contributing the total flux density, cf. Figure 1.)

accretion disk seed photons and/or BLR seed photons. The bulk Lorentz factor of the jet and the spectral shape of the electron distribution are varied until an acceptable fit to the observed optical to gamma spectrum is found.

From the previous discussion the weak point of this approach is apparent: the flux variations are assumed to be due to changes of the flow, while in reality the luminosity of the flow remains unchanged and the variations - as well as most of the total flux during active phases - originate in the shocks. For 3C 279 we can even estimate the relative contributions of the various components, since it was one of the blazars monitored intensively between 22 and $375 \mathrm{GHz}$ during 1988-1994 with JCMT, SEST, and Metsähovi telescopes (Stevens et al. 1994). From these data we can derive the approximate spectral shape of the underlying jet (i.e. quiescent flux levels at the various frequencies) as well as of the shocks during the EGRET epochs. The underlying jet component contributes appreciably to the IR to optical flux only during the most quiet stages of 3C 279 when hardly any gamma rays are detected. When 3C 279 is flaring, over $90 \%$ of the IR peak flux comes from the newly created shock. As an example, Figure 3 shows the estimated jet (constant) and the shock (changing) components during a moderate flare in 1991.0, compared to the jet component in 1991.47 from Hartman et al. (2001). It is obvious that the shocks must dominate the gamma-ray production.

There is also other evidence linking shocks to gammaray emission. There is no conceivable mechanism by which one could change the jet flow parameters (the bulk Lorentz factor!) rapidly enough to explain the gammaray timescales of weeks or even days. In contrast, the shock variability timescales appear to be suitable. Using the Stevens et al. (1994) data we can estimate the decrease in the flare variability timescales from 22 to $375 \mathrm{GHz}$. 


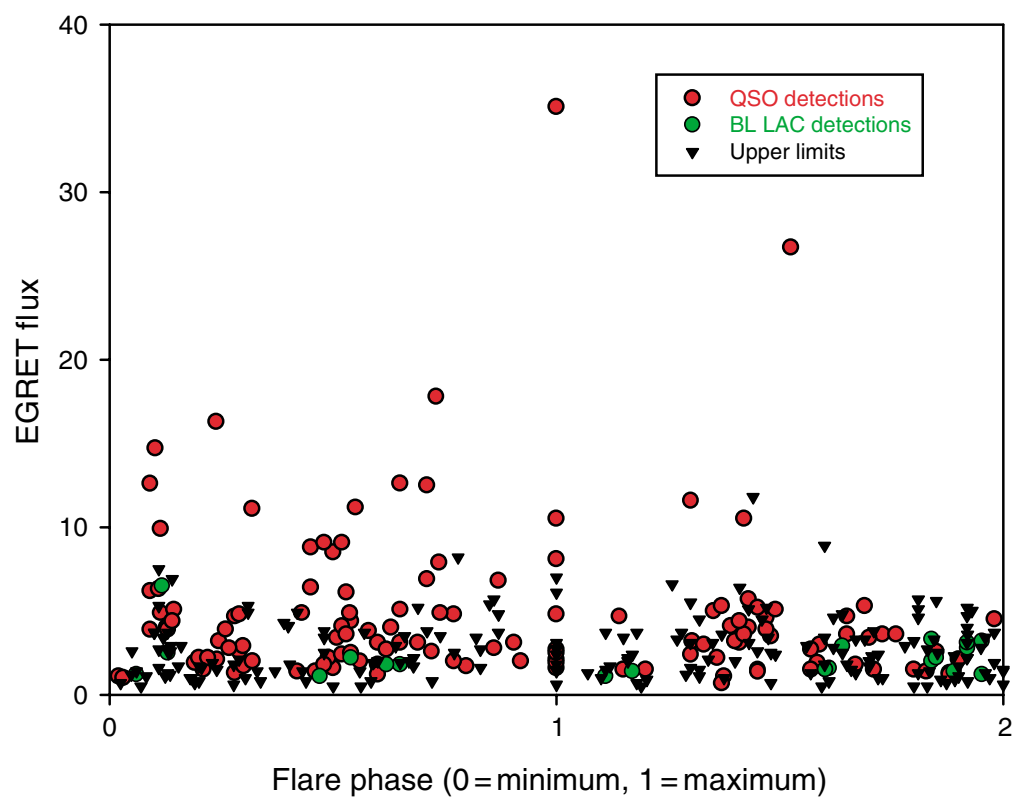

Figure 4 All the EGRET pointings towards sources included in the Metsähovi monitoring program. The strong QSO gamma flares occur mainly during the rise of the radio flare, while the weak detections seem to be independent of the radio phase. Note that no strong BL Lac gamma flares have been detected, and that there is no correlation with the radio flare phase in their case.

Extrapolating to $10^{13} \mathrm{~Hz}$ (where the major contribution to gamma rays presumably originates) gives a timescale of about a week. Even more revealing is the well-established statistical correlation between radio and gamma-ray flares: strong EGRET detections occur preferentially during the rise or peak of millimetre flares (Valtaoja \& Teräsranta 1995, 1996), as expected if the gamma rays come from the shocks. Figure 4 shows an updated comparison using the full EGRET and Metsähovi data.

All the evidence indicates that gamma rays originate in the shocks, not in the innermost portions of the jet. Thus, if one wants to use one-component models for the gammaray emission, that component should be the thin, rather homogeneous shock and not the inhomogeneous jet. In a more realistic case one should have both the shock (or often, several shocks) and the jet included in the model calculations.

\section{External Compton?}

Finally, our results cast grave doubts on the external Compton models for gamma-ray production. To see this, consider Figure 4. The largest amounts of gamma rays are emitted after the TFD flare has begun, after the VLBI component has been ejected. We have calculated the time from the beginning of the radio flare, $t_{\max }-\tau$, to the EGRET gamma-ray flare for each of the strongest EGRET flares (over 8 units). The average $\Delta t$ is $67 \pm 83$ days, virtually the same as obtained by Jorstad et al. (2001b) by comparing the gamma-ray flare epochs to the VLBI zero epochs ( $52 \pm 76$ days) and to the minima in $14.5 \mathrm{GHz}$ polarised flux ( $69 \pm 68$ days). Thus, three different methods give the same result: on the average, the gamma-ray flare occurs about two months after the formation of the shock. In this observed time interval the shock has moved down the jet an average distance $\Delta L=(\Gamma D \beta c \Delta t) /(1+z) \approx 1.5 \mathrm{pc}$ (using $\Gamma$ and D from Lähteenmäki \& Valtaoja 1999)

Most EC models propose that the seed photons come either from the accretion disk or are reflected from the BLR. This is possible only if the gamma rays are coming from a component very close to the accretion disk or at least inside the BLR. All the observational evidence indicates that this is not the case. In the particular case of 3C 279, the two strongest flares in 1991.47 and 1996.09 (Figure 1) occurred 0.5-0.7 years after the onsets of the TFD flares (in 1990.68 and 1995.55, as determined from our monitoring data) and the ejections of the VLBI components (in 1990.88 and 1995.63, Wehrle et al. 2001). With $D \approx 15$ and $\Gamma \approx 9$ (Lähteenmäki \& Valtaoja 1999; Hartman et al. 2001), this translates into a distance of 15-20 pc down the jet. In comparison, the BLR ends at $0.4 \mathrm{pc}$ in the Hartman et al. (2001) model fits.

The EC mechanism may be important in some sources, in particular those where a part of the gamma radiation does originate in the jet flow (see below). However, in the case of strong gamma-ray flares, coming from shocks far downstream, disk or cloud photons cannot be the seed population. It may be possible that Compton reflection or a torus around the AGN could produce sufficient amounts of seed photons for the EC mechanism to be important; such models deserve more attention.

\section{Towards Understanding Shocked Jet Physics}

Comparisons between radio data, synchrotron/IC spectra, and EGRET gamma-ray data are yielding some hints about the physics of shocked jets, but clear answers must await the next generation of gamma-ray telescopes. 
For example, the radio/gamma-ray connections are different for quasars and for BL Lacs. Strong gamma-ray detections come from quasars during a rising mm-flare (Figure 4); BL Lacs are relatively weak emitters, and the detection does not appear to be related to the radio flares. This might indicate that the (jet?) EC mechanism is important in BL Lacs, and the shocks play a smaller role than in quasars.

We have also compared the Lorentz factors (from Lähteenmäki \& Valtaoja 1999), the synchrotron peak frequencies (Ghisellini et al. 1998), the absolute radio luminosity, and the Compton dominance $S_{\gamma} / S_{\text {radio }}$ in our quasar sample. We find that the flows in high synchrotron peak quasars tend to be slower, more powerful, and more IC-dominated. This is opposite to the trends found when quasars and BL Lacs (FR II and FR I jets!) are lumped together into a single 'blazar' population.

There also seems to be a trend in the gamma-ray and radio variability patterns: sources with low synchrotron peak frequency (e.g. 3C 273) tend to have large radio variations and an almost constant gamma-ray flux, while sources with high peak frequencies (e.g. 0954+55) have large gamma-ray variations and little associated radio variability. However, as we at present do not have any viable models for gamma-ray production, it is difficult to interpret these results.

\section{Conclusions}

All radio to IR variations in AGN are due to shocks growing and decaying in the approximately constant underlying jet; there is no evidence that the flow parameters - or synchrotron/IC radiation from the flow - changes significantly in short timescales. Since much of the shock growth and decay occurs within the innermost few tenths of a milliarcsecond, present day VLBI may lead one to the erroneous conclusion that variations are coming from the jet/core and not from the shocks.

Consequently, shocks must produce the strongest gamma-ray flares. This is also indicated by the spectra of the radio to IR components, the timescales, and the time delays. On the average, the shocks are over a parsec downstream from the radio core at the time of the gamma-ray flare, beyond significant disk or BLR photon fields. If external Compton mechanisms are important, the seed photons must come from elsewhere. Realistic two-component (shock and jet) models with realistic parameters are urgently needed in order to understand the gamma-ray production in shocked jets and the physics of relativistic flows in general.

\section{Acknowledgments}

We are grateful for Svetlana Jorstad and Alan Marscher for providing the VLBI monitoring data prior to publication.

\section{References}

Abraham, Z., Carrara, E. A., Zensus, J. A., \& Unwin, S. C. 1996, A\&AS, 115, 543

Böttcher, M., \& Bloom, S. D. 2000, AJ, 119, 469

Britzen, S., Witzel, A., Krichbaum, T. P., Campbell, R. M., Wagner, S. J., \& Qian, S. J. 2000, A\&A, 360, 65

Ghisellini, G., Celotti, A., Fossati, G., Maraschi, L., \& Comastri, A. 1998, MNRAS, 301, 451

Hartman, R. C., et al. 2001, ApJ, 553, 683

Hughes, P. A., Aller, H. D., \& Aller, M. F. 1985, ApJ, 298, 301

Jorstad, S. G., Marscher, A. P., Mattox, J. R., Wehrle, A. E., Bloom, S. D., \& Yurchenko, A. V. 2001a, ApJS, 134, 181

Jorstad, S. G., Marscher, A. P., Mattox, J. R., Aller, M. F., Aller, H. D., Wehrle, A. E., \& Bloom, S. D. 2001b, ApJ, 556, 738

Krichbaum, T. P., Krauss, A., Otterbein, K., Britzen, S., Witzel, A., \& Zensus, J. A. 1998, in Radio Emission from Galactic and Extragalactic Compact Sources, IAU Colloquium 164, eds J. A. Zensus, G. B. Taylor, \& J. B. Wrobel (San Francisco: ASP), 37

Lähteenmäki, A., \& Valtaoja, E. 1999, ApJ, 521, 493

Marscher, A. P., \& Gear, W. K. 1985, ApJ, 298, 114

Stevens, J. A., Litchfield, S. J., Robson, E. I., Hughes, D. H., Gear, W. K., Teräsranta, H., Valtaoja, E., \& Tornikoski, M. 1994, ApJ, 437, 91

Teräsranta, H., et al. 1998, A\&AS, 132, 305

Türler, M., Courvoisier, T. J.-L., \& Paltani, S. 1999, A\&A, 349,45

Valtaoja, E., \& Teräsranta, H. 1995, A\&A, 297, L13

Valtaoja, E., \& Teräsranta, H. 1996, A\&AS, 120, 491

Valtaoja, E., Lähteenmäki, A., Teräsranta, H., \& Lainela, M. 1999, ApJS, 120, 95

Valtaoja, E., Teräsranta, H., Urpo, S., Nesterov, N. S., Lainela, M., \& Valtonen, M. 1992, A\&A, 254, 71

Valtaoja, E., et al. 1988, A\&A, 203, 1

Wehrle, A. E., Piner, B. G., Unwin, S. C., Zook, A. C., Xu, W., Marscher, A. P., Teräsranta, H., \& Valtaoja, E. 2001, ApJS, 133,297 